\title{
Solvability for a fractional $p$-Laplacian multipoint boundary value problem at resonance on infinite interval
}

\author{
Wei Zhang, Wenbin Liu* and Taiyong Chen
}

\section{"Correspondence:} wblium@163.com

Department of Mathematics, China University of Mining and

Technology, Xuzhou, 221116, P.R. China

\section{照 Springer}

\begin{abstract}
In this paper, we study the multipoint boundary value problem for a fractional $p$-Laplacian equation at resonance on infinite interval and establish the existence result of solutions by using extension of Mawhin's continuation theorem. Our paper enriches some known existing articles. In order to illustrate our main result, we give an example.
\end{abstract}

MSC: $34 \mathrm{~A} 08 ; 34 \mathrm{~B} 15$

Keywords: boundary value problem; fractional $p$-Laplacian equation; resonance; infinite interval; continuation theorem

\section{Introduction}

Many boundary value problems (BVPs) on infinite interval arise naturally in the study of radially symmetric solutions of nonlinear elliptic equations (see [1]). There are numerous physical models giving us motivations to investigate BVPs on infinite interval, such as the following two important examples.

The first model is for determining the electric potential in an isolated atom derived independently by Thomas and Fermi:

$$
\left\{\begin{array}{l}
u^{\prime \prime}(t)=t^{-1 / 2} u^{3 / 2} \\
u(0)=1, \quad u(\infty)=0
\end{array}\right.
$$

Another famous model is the well-known Blasius boundary layer equation that shows flow past a semiinfinite flat plate in hydromechanics:

$$
\left\{\begin{array}{l}
f^{\prime \prime \prime}(\eta)+\frac{1}{2} f(\eta) f^{\prime \prime}(\eta)=0, \quad \eta \in[0,+\infty), \\
f(0)=f^{\prime}(0)=0, \quad f^{\prime}(\infty)=1 .
\end{array}\right.
$$

Although the Blasius equation is simple, it can clearly reveal the essence of the problems, which is convenient for people to carry out theoretic analysis and mathematical research for boundary layer problem. So, BVPs on infinite interval have important significance and have been received much attention (see [1]). There are a large number of papers discussing

(c) 2016 Zhang et al. This article is distributed under the terms of the Creative Commons Attribution 4.0 International License (http://creativecommons.org/licenses/by/4.0/), which permits unrestricted use, distribution, and reproduction in any medium, provided you give appropriate credit to the original author(s) and the source, provide a link to the Creative Commons license, and indicate if changes were made. 
the existence of solutions for both integral-order and fractional-order differential BVPs on infinite interval by using the techniques of nonlinear analysis such as variational method (see [2]), fixed-point theorems (see [3-8]), upper and lower solutions method (see [9, 10]), fixed-point index theory (see [11,12]), coincidence degree theory (see [13-15]), etc.

Jiang [13] proved the existence of solutions for the $p$-Laplacian boundary value problem

$$
\left\{\begin{array}{l}
\left(\phi_{p}\left(u^{\prime}(t)\right)\right)^{\prime}+f\left(t, u, u^{\prime}\right)=0, \quad 0<t<+\infty \\
u(0)=0, \quad \phi_{p}\left(u^{\prime}(\infty)\right)=\sum_{i=1}^{n} \alpha_{i} \phi_{p}\left(u^{\prime}\left(\xi_{i}\right)\right)
\end{array}\right.
$$

where $\phi_{p}(s)=|s|^{p-2} s, \phi_{p}(0)=0, p>1$. The main method of the paper was the coincidence degree theory.

Su and Zhang [6] investigated the existence of unbounded solutions of boundary value problem

$$
\left\{\begin{array}{l}
D_{0+}^{\alpha} u(t)=f\left(t, u(t), D_{0+}^{\alpha-1} u(t)\right), \quad t \in J:=[0, \infty) \\
u(0)=0, \quad D_{0+}^{\alpha-1} u(+\infty)=u_{\infty}, \quad u_{\infty} \in \mathbb{R}
\end{array}\right.
$$

where $D_{0+}^{\alpha}$ and $D_{0+}^{\alpha-1}$ are the standard Riemann-Liouville fractional derivatives of order $1<\alpha \leq 2$. The main result of this paper was obtained by using Schauder's fixed point theorem.

Zhou, Kou, and Xie (see [15]) studied the existence of solutions for the following multipoint boundary value problem:

$$
\left\{\begin{array}{l}
D_{0+}^{\alpha} x(t)=f\left(t, x(t), D_{0+}^{\alpha-1} x(t)\right), \quad t \in(0, \infty), \\
x(0)=0, \quad \lim _{t \rightarrow+\infty} D_{0+}^{\alpha-1} x(t)=\beta x(\eta)
\end{array}\right.
$$

where $D_{0_{+}}^{\alpha}$ is the standard Riemann-Liouville fractional derivative of order $\left.1<\alpha \leq 2, \eta\right\rangle 0$. The analysis of this paper relied on the coincidence degree of Mawhin.

Motivated by the results mentioned, in this paper, we use the extension of Mawhin's continuation theorem (see [16]) to discuss the existence of solutions for the following multipoint boundary value problem of fractional $p$-Laplacian equation at resonance:

$$
\left\{\begin{array}{l}
\left(\phi_{p}\left(D_{0+}^{\alpha} x(t)\right)\right)^{\prime}+f\left(t, x(t), D_{0+}^{\alpha-1} x(t), D_{0+}^{\alpha} x(t)\right)=0, \quad 0<t<+\infty \\
x(0)=x^{\prime}(0)=0, \quad \phi_{p}\left(D_{0+}^{\alpha} x(+\infty)\right)=\sum_{i=1}^{n} \alpha_{i} \phi_{p}\left(D_{0+}^{\alpha} x\left(\xi_{i}\right)\right)
\end{array}\right.
$$

where $1<\alpha \leq 2, D_{0+}^{\alpha}$ is the standard Riemann-Liouville fractional derivative, $0<\xi_{1}<\xi_{2}<$ $\cdots<\xi_{n}<+\infty, \alpha_{i}>0, \sum_{i=1}^{n} \alpha_{i}=1, \phi_{p}$ is reversible, and by $\phi_{q}$ we denote the inverse operator of $\phi_{p}$, where $1 / p+1 / q=1$.

As we know, fractional differential equations have been applied in various fields (see [17, 18]). So, it is meaningful to discuss the boundary value problems of fractional differential equations on infinite interval.

Throughout this paper, we suppose that the following hypothesis is satisfied:

$\left(A_{1}\right) f \in C[0,+\infty) \times \mathbb{R}^{3} \rightarrow \mathbb{R}$ is an $L^{1}$-Caratheodory function, that is, $f$ is a Caratheodory function, and for any $r>0$, there exists a nonnegative function $g_{r}(t) \in L^{1}[0,+\infty)$ such 
that

$$
|f(t, u, v, w)| \leq g_{r}(t), \quad \text { a.e. } t \in[0,+\infty), u, v, w \in \mathbb{R},\|u\| \leq r,\|v\| \leq r,\|w\| \leq r .
$$

\section{Preliminaries}

In this section, we introduce some definitions and lemmas.

Definition 2.1 (See $[19,20])$ The Rieman-Liouville fractional integral of order $\alpha>0$ for a function $u:(0,+\infty) \rightarrow \mathbb{R}$ is defined as

$$
I_{0+}^{\alpha} u(t)=\frac{1}{\Gamma(\alpha)} \int_{0}^{t}(t-s)^{\alpha-1} u(s) d s
$$

where $\Gamma(\alpha)$ is the gamma function, provided that the right-hand side is pointwise defined on $(0,+\infty)$.

Definition 2.2 (See $[19,20]$ ) The Riemann-Liouville functional derivative of order $\alpha>0$ for a function $u:(0,+\infty) \rightarrow \mathbb{R}$ is defined as

$$
D_{0+}^{\alpha} u(t)=\frac{d^{n}}{d t^{n}} I_{0+}^{n-\alpha} u(t)=\frac{1}{\Gamma(n-\alpha)} \frac{d^{n}}{d t^{n}} \int_{0}^{t}(t-s)^{n-\alpha-1} u(s) d s,
$$

where $n=[\alpha]+1$, provided that the right-hand side is pointwise defined on $(0,+\infty)$.

Lemma 2.1 (See $[8,15])$ Assume that $u \in C[0, \infty) \cap L(0, \infty)$ with a fractional derivative of order $\alpha>0$ that belongs to $C[0, \infty) \cap L(0, \infty)$. Then

$$
I_{0+}^{\alpha} D_{0+}^{\alpha} u(t)=u(t)+c_{1} t^{\alpha-1}+c_{2} t^{\alpha-2}+\cdots+c_{n} t^{\alpha-n}
$$

for some $c_{i} \in R, i=1,2, \ldots, n, n=[\alpha]+1$.

Definition 2.3 (See $[13,16]$ ) Let $X$ and $Y$ be two Banach spaces with norms $\|\cdot\|_{X}$ and $\|\cdot\|_{Y}$, respectively. A continuous operator $M: X \cap \operatorname{dom} M \rightarrow Y$ is said to be quasi-linear if

(a) $\operatorname{Im} M:=M(X \cap \operatorname{dom} M)$ is a closed subset of $Y$, and

(b) $\operatorname{Ker} M:=\{x \in X \cap \operatorname{dom} M: M x=0\}$ is linearly homeomorphic to $\mathbb{R}^{n}, n<\infty$.

Take $X_{1}=\operatorname{Ker} M$ and let $X_{2}$ be the complement space of $X_{1}$ in $X$, so that, $X=X_{1} \oplus X_{2}$. On the other hand, suppose that $Y_{1}$ is a subspace of $Y$ and $Y_{2}$ is the complement space of $Y_{1}$ in $Y$, so that $Y=\mathrm{Y}_{1} \oplus Y_{2}$. Let $P: X \rightarrow X_{1}$ and $Q: Y \rightarrow Y_{1}$ be two projectors, and $\Omega \subset X$ be an open bounded set with the origin $\theta \in \Omega$. Throughout we use $\theta$ to denote the origin in a linear space (see $[13,16])$.

Definition 2.4 (See $[13,16]$ ) Suppose that $N_{\lambda}: \bar{\Omega} \rightarrow Y, \lambda \in[0,1]$, is a continuous operator. Denote $N_{1}$ by $N$. Let $\sum_{\lambda}=\left\{x \in \bar{\Omega}: M x=N_{\lambda} x\right\}$. $N_{\lambda}$ is said to be $M$-compact in $\bar{\Omega}$ if there is a vector subspace $Y_{1}$ of $Y$ with $\operatorname{dim} Y_{1}=\operatorname{dim} X_{1}$ and a continuous and compact operator $R: \bar{\Omega} \times[0,1] \rightarrow X_{2}$ such that, for $\lambda \in[0,1]$,

$\left(a_{1}\right)(I-Q) N_{\lambda}(\bar{\Omega}) \subset \operatorname{Im} M \subset(I-Q) Y$;

$\left(a_{2}\right) Q N_{\lambda} x=\theta, \lambda \in(0,1) \Leftrightarrow Q N x=\theta$; 
(a) $R(\cdot, 0)$ is the zero operator, and $\left.R(\cdot, \lambda)\right|_{\sum_{\lambda}}=\left.(I-P)\right|_{\sum_{\lambda}}$;

$\left(a_{4}\right) M[P+R(\cdot, \lambda)]=(I-Q) N_{\lambda}$.

Lemma 2.2 (Extension of Mawhin's continuation theorem) Let $X$ and $Y$ be two Banach spaces with the norms $\|\cdot\|_{X}$ and $\|\cdot\|_{Y}$ respectively, and $\Omega \subset X$ be an open bounded nonempty set. Suppose that

$$
M: X \cap \operatorname{dom} M \rightarrow Y
$$

is a quasi-linear operator and

$$
N_{\lambda}: \bar{\Omega} \rightarrow Y, \quad \lambda \in[0,1],
$$

is $M$-compact on $\bar{\Omega}$. In addition, let the following conditions hold:

$\left(B_{1}\right) M x \neq N_{\lambda} x, \forall(x, \lambda) \in \partial \Omega \times(0,1)$,

$\left(B_{2}\right) Q N x \neq 0, \forall x \in \operatorname{Ker} M \cap \partial \Omega$,

$\left(B_{3}\right) \operatorname{deg}(J Q N, \operatorname{Ker} M \cap \Omega, 0) \neq 0$.

Then the abstract equation $M x=N x$ has at least one solution in $\operatorname{dom} M \cap \bar{\Omega}$, where $N=N_{1}$, $Q: Y \rightarrow \operatorname{Im} Q$ is a projector, $J: \operatorname{Im} Q \rightarrow \operatorname{Ker} M$ is a homeomorphism with $J(\theta)=\theta($ see $[16])$.

\section{Main result}

In this section, we give a theorem on the existence of solutions for BVP (1.1). Let

$$
\begin{aligned}
& X=\left\{x \mid x, D_{0+}^{\alpha} x \in C[0,+\infty), \sup _{t \in[0,+\infty)} \frac{|x(t)|}{1+t^{\alpha}}<+\infty,\right. \\
& \left.\sup _{t \in[0,+\infty)} \frac{\left|D_{0+}^{\alpha-1} x(t)\right|}{1+t^{\alpha}}<+\infty, \sup _{t \in[0,+\infty)}\left|D_{0+}^{\alpha} x(t)\right|<+\infty\right\}, \\
& Y=L^{1}[0,+\infty),
\end{aligned}
$$

with norms

$$
\|x\|_{X}=\max \left\{\|x\|_{0},\left\|D_{0+}^{\alpha-1} x\right\|_{0},\left\|D_{0+}^{\alpha} x\right\|_{\infty}\right\}, \quad\|y\|_{Y}=\|y\|_{1},
$$

where $\|y\|_{1}=\int_{0}^{+\infty}|y(t)| d t,\left\|D_{0_{+}}^{\alpha} x\right\|_{\infty}=\sup _{t \in[0,+\infty)}\left|D_{0+}^{\alpha} x(t)\right|,\|x\|_{0}=\left\|\frac{x(t)}{1+t^{\alpha}}\right\|_{\infty}$. Clearly, $\left(X,\|\cdot\|_{X}\right)$ and $\left(Y,\|\cdot\|_{Y}\right)$ are Banach spaces.

Define the operators $M: \operatorname{dom} M \subset X \rightarrow Y$ and $N_{\lambda}: X \rightarrow Y$ as follows:

$$
M x=\left(\phi_{p}\left(D_{0+}^{\alpha} x\right)\right)^{\prime}, \quad N_{\lambda} x(t)=-\lambda f\left(t, x(t), D_{0+}^{\alpha-1} x(t), D_{0+}^{\alpha} x(t)\right), \quad \lambda \in[0,1], x \in X,
$$

where

$$
\begin{aligned}
\operatorname{dom} M= & \left\{x \in X \mid \phi_{p}\left(D_{0+}^{\alpha} x\right) \in A C[0,+\infty), x(0)=x^{\prime}(0)=0,\right. \\
& \left.\phi_{p}\left(D_{0+}^{\alpha} x(+\infty)\right)=\sum_{i=1}^{n} \alpha_{i} \phi_{p}\left(D_{0+}^{\alpha} x\left(\xi_{i}\right)\right)\right\} .
\end{aligned}
$$

Then the BVP (1.1) is equivalent to $M x=N x, x \in \operatorname{dom} M$. 
Lemma 3.1 For $M$ defined as before, we have

$$
\begin{aligned}
& \operatorname{Ker} M=\left\{x \in \operatorname{dom} M \mid x(t)=c t^{\alpha}, \forall t \in[0,+\infty), c \in \mathbb{R}\right\} \\
& \operatorname{Im} M=\left\{y \in Y \mid \sum_{i=1}^{n} \alpha_{i} \int_{\xi_{i}}^{+\infty} y(s) d s=0\right\}
\end{aligned}
$$

and $M$ is a quasi-linear operator.

Proof For $x \in \operatorname{Ker} M, M x=0$, that is, $\left(\phi_{p}\left(D_{0_{+}}^{\alpha} x\right)\right)^{\prime}=0$, by $\phi_{p}\left(D_{0_{+}}^{\alpha} x(+\infty)\right)=\sum_{i=1}^{n} \alpha_{i} \times$ $\phi_{p}\left(D_{0+}^{\alpha} x\left(\xi_{i}\right)\right)$ we easily get

$$
\phi_{p}\left(D_{0+}^{\alpha} x(t)\right)=\phi_{p}\left(D_{0+}^{\alpha} x(+\infty)\right)=\sum_{i=1}^{n} \alpha_{i} \phi_{p}\left(D_{0+}^{\alpha} x\left(\xi_{i}\right)\right) .
$$

Based on Lemma 2.1, since $x(0)=x^{\prime}(0)=0$, we have

$$
\begin{aligned}
x(t) & =c_{1} t^{\alpha-1}+c_{2} t^{\alpha-2}+\frac{\phi_{q}\left(\sum_{i=1}^{n} \alpha_{i} \phi_{p}\left(D_{0+}^{\alpha} x\left(\xi_{i}\right)\right)\right)}{\Gamma(\alpha)} \int_{0}^{t}(t-s)^{\alpha-1} d s \\
& =\frac{\phi_{q}\left(\sum_{i=1}^{n} \alpha_{i} \phi_{p}\left(D_{0_{+}}^{\alpha} x\left(\xi_{i}\right)\right)\right)}{\Gamma(\alpha+1)} t^{\alpha} .
\end{aligned}
$$

Conversely, if $x=c t^{\alpha}$, then $M x=0$ by (3.1). If $y \in \operatorname{Im} M$, then there exists a function $x \in$ $\operatorname{dom} M$ such that $y(t)=\left(\phi_{p}\left(D_{0+}^{\alpha} x(t)\right)\right)^{\prime}$. Then

$$
\begin{aligned}
\phi_{p}\left(D_{0+}^{\alpha} x(t)\right) & =\phi_{p}\left(D_{0+}^{\alpha} x(+\infty)\right)-\int_{t}^{+\infty} y(s) d s \\
& =\sum_{i=1}^{n} \alpha_{i} \phi_{p}\left(D_{0+}^{\alpha} x\left(\xi_{i}\right)\right)-\int_{t}^{+\infty} y(s) d s \\
& =\sum_{i=1}^{n} \alpha_{i}\left[\phi_{p}\left(D_{0+}^{\alpha} x(+\infty)\right)-\int_{\xi_{i}}^{+\infty} y(s) d s\right]-\int_{t}^{+\infty} y(s) d s,
\end{aligned}
$$

that is,

$$
\sum_{i=1}^{n} \alpha_{i} \int_{\xi_{i}}^{+\infty} y(s) d s=0
$$

On the other hand, if $y \in Y$ satisfies (3.3), then take

$$
x(t)=I_{0+}^{\alpha} \phi_{q}\left(-\int_{t}^{+\infty} y(s) d s\right) .
$$

Then $x \in \operatorname{dom} M$ and $M x=y$. Hence, (3.2) holds. Clearly, $\operatorname{dim} \operatorname{Ker} M=1<+\infty$ and $\operatorname{Im} M:=M(\operatorname{dom} M \cap X)$ is a closed subset of $Y$. Therefore, we get that $M$ is a quasi-linear operator.

Lemma 3.2 (See [1]) Let $V \subset C_{\infty}=\left\{u \in C[0, \infty), \lim _{t \rightarrow+\infty} u(t)\right.$ exists $\}$. Then $V$ is relatively compact if the following conditions hold: 
$\left(b_{1}\right)$ all functions from $V$ are uniformly bounded,

$\left(b_{2}\right)$ all functions from $V$ are equicontinuous on any compact interval of $[0,+\infty)$,

$\left(b_{3}\right)$ all functions from $V$ are equiconvergent at infinity.

Remark 3.1 By Lemma 3.2, any set $V \subset X$ (defined as before) is relatively compact, we only need to show that the sets

$$
V_{1}=\left\{\frac{x(t)}{1+t^{\alpha}} \mid x \in V\right\}, \quad V_{2}=\left\{\frac{D_{0+}^{\alpha-1} x(t)}{1+t^{\alpha}} \mid x \in V\right\}, \quad V_{3}=\left\{D_{0+}^{\alpha} x(t) \mid x \in V\right\}
$$

are uniformly bounded in $X$, equicontinuous on any compact intervals of $[0,+\infty)$, and equiconvergent at infinity.

Lemma 3.3 Let $\Omega \subset X$ be nonempty, open, and bounded. Then $N_{\lambda}$ is M-compact on $\bar{\Omega}$.

Proof Define two projectors $P: X \rightarrow X_{1}$ and $Q: Y \rightarrow Y_{1}$ by

$$
P x(t)=\frac{D_{0+}^{\alpha} x(+\infty)}{\Gamma(\alpha+1)} t^{\alpha}, \quad Q y(t)=\frac{\sum_{i=1}^{n} \alpha_{i} \int_{\xi_{i}}^{+\infty} y(s) d s}{\sum_{i=1}^{n} \alpha_{i} e^{-\xi_{i}}} e^{-t}, \quad \forall t \in[0,+\infty),
$$

where $X_{1}=\operatorname{Ker} M$ and $Y_{1}=\operatorname{Im} Q$.

First, we show that $\left(a_{1}\right)$ and $\left(a_{2}\right)$ in Definition 2.4 hold. In fact, by the definition of $P$ we get $\operatorname{Im} P=\operatorname{Ker} M$ and $P^{2} x(t)=P x(t)$. For $x \in X$, since $x=(x-P x)+P x$ and $\operatorname{Im} P=\operatorname{Ker} M$, we have $(x-P x) \in \operatorname{Ker} P, P x \in \operatorname{Ker} M$. We easily see that $\operatorname{Ker} M \cap \operatorname{Ker} P=\{0\}$. So, $X=$ $\operatorname{Ker} M \oplus \operatorname{Ker} P=X_{1} \oplus X_{2}$. Similarly, by the definition of $Q$, we can obtain

$$
Q^{2} y=Q(Q y)=\frac{\sum_{i=1}^{n} \alpha_{i} \int_{\xi_{i}}^{+\infty} Q y d s}{\sum_{i=1}^{n} \alpha_{i} e^{-\xi_{i}}} e^{-t}=Q y
$$

and $\operatorname{Ker} Q=\operatorname{Im} M$. For $y \in Y$, since $y=(y-Q y)+Q y$ and $\operatorname{Ker} Q=\operatorname{Im} M$, we have $(y-Q y) \in$ $\operatorname{Ker} Q, Q y \in \operatorname{Im} M$. Clearly, $\operatorname{Im} Q \cap \operatorname{Im} M=\{0\}$. So, we have $Y=\operatorname{Im} Q \oplus \operatorname{Im} M=Y_{1} \oplus Y_{2}$ and $\operatorname{dim} X_{1}=\operatorname{dim} \operatorname{Ker} M=\operatorname{dim} \operatorname{Im} Q=\operatorname{dim} Y_{1}$, where $X_{2}=\operatorname{Ker} P, Y_{2}=\operatorname{Im} M$. Let $\Omega \subset X$ be open bounded, and let $\theta \in \Omega$. On the one hand, for $x \in \bar{\Omega}$, since $Q(I-Q)$ is a zero operator, we have $Q\left[(I-Q) N_{\lambda} x\right]=0$; thus, $(I-Q) N_{\lambda} x \in \operatorname{Ker} Q=\operatorname{Im} M$, that is, $(I-Q) N_{\lambda}(\bar{\Omega}) \subset \operatorname{Im} M$. On the other hand, for $y \in \operatorname{Im} M$, since $y=(y-Q y)+Q y$ and $\operatorname{Ker} Q=\operatorname{Im} M$, we have $y \in(I-Q) Y$, that is, $\operatorname{Im} M \subset(I-Q) Y$. Clearly, $Q N_{\lambda} x=0, \lambda \in(0,1) \Leftrightarrow Q N x=0$. So, conditions $\left(a_{1}\right)$ and $\left(a_{2}\right)$ of Definition 2.4 hold.

Second, we give the definition of operator $R$ and aim to show that $R$ is compact. For notational convenience, let

$$
l(t, x, \lambda)=\int_{t}^{+\infty}(Q-I) N_{\lambda} x(s) d s+\phi_{p}\left(D_{0+}^{\alpha} x(+\infty)\right), h(t)=\phi_{q}(l(t, x, \lambda))-D_{0+}^{\alpha} x(+\infty) .
$$

Define the operator $R: \bar{\Omega} \times[0,1] \rightarrow X_{2}$ by

$$
R(x, \lambda)(t)=I_{0+}^{\alpha} h(t) .
$$

By $\left(A_{1}\right)$ it is easy to know that $R(x, \lambda)(t)$ is continuous on $\bar{\Omega} \times[0,1]$. 
Step 1. We prove that $R(x, \lambda)(\bar{\Omega})$ is both uniformly bounded in $X$ and equicontinuous on any compact interval of $[0,+\infty)$. In fact, since $\Omega \subset X$ is nonempty, open, and bounded, by $\left(A_{1}\right)$ there exist a constant $r>0$ and a nonnegative function $g_{r}(t) \in L^{1}[0,+\infty)$ such that

$$
\|x\|_{X} \leq r, \quad\left|f\left(t, x(t), D_{0+}^{\alpha-1} x(t), D_{0_{+}}^{\alpha} x(t)\right)\right| \leq g_{r}(t), \quad \text { a.e. } t \in[0,+\infty), x \in \bar{\Omega} .
$$

Since

$$
\begin{aligned}
\left|\int_{s}^{+\infty}(Q-I) N_{\lambda} x d \tau\right| & \\
\leq & \int_{s}^{+\infty}\left|Q N_{\lambda} x-N_{\lambda} x\right| d \tau \\
\leq & \int_{0}^{+\infty} \mid f\left(\tau, x(\tau), D_{0+}^{\alpha-1} x(\tau), D_{0+}^{\alpha} x(\tau)\right) \\
& \quad-\frac{\sum_{i=1}^{n} \alpha_{i} \int_{\xi_{i}}^{+\infty} f\left(s, x(s), D_{0+}^{\alpha-1} x(s), D_{0+}^{\alpha} x(s)\right) d s}{\sum_{i=1}^{n} \alpha_{i} e^{-\xi_{i}}} e^{-\tau} \mid d \tau \\
\leq & \int_{0}^{+\infty}\left|f\left(\tau, x(\tau), D_{0+}^{\alpha-1} x(\tau), D_{0+}^{\alpha} x(\tau)\right)\right| d \tau \\
& +\left|\frac{\sum_{i=1}^{n} \alpha_{i} \int_{\xi_{i}}^{+\infty} f\left(s, x(s), D_{0+}^{\alpha-1} x(s), D_{0+}^{\alpha} x(s)\right) d s}{\sum_{i=1}^{n} \alpha_{i} e^{-\xi_{i}}}\right| \\
\leq & \left\|g_{r}\right\|_{1}\left(1+\frac{1}{\sum_{i=1}^{n} \alpha_{i} e^{-\xi_{i}}}\right)=: \tilde{r},
\end{aligned}
$$

we have

$$
\begin{aligned}
|h(s)| & =\left|\phi_{q}\left[\int_{s}^{+\infty}(Q-I) N_{\lambda} x(\tau) d \tau+\phi_{p}\left(D_{0+}^{\alpha} x(+\infty)\right)\right]-D_{0+}^{\alpha} x(+\infty)\right| \\
& \leq \phi_{q}\left[\tilde{r}+\phi_{p}(r)\right]+r=: m .
\end{aligned}
$$

Therefore, for any $x \in \bar{\Omega}$, we have

$$
\begin{aligned}
& \|R(x, \lambda)(t)\|_{0}=\sup _{t \in[0,+\infty)}\left|\frac{R(x, \lambda)(t)}{1+t^{\alpha}}\right| \\
& =\sup _{t \in[0,+\infty)} \frac{1}{\Gamma(\alpha)}\left|\int_{0}^{t} \frac{(t-s)^{\alpha-1}}{1+t^{\alpha}} h(s) d s\right| \\
& \leq \frac{m}{\Gamma(\alpha)} \sup _{t \in[0,+\infty)} \int_{0}^{t} \frac{(t-s)^{\alpha-1}}{1+t^{\alpha}} d s \\
& \leq \frac{m}{\Gamma(\alpha+1)} \leq m, \\
& \left\|D_{0+}^{\alpha-1} R(x, \lambda)(t)\right\|_{0}=\sup _{t \in[0,+\infty)}\left|\frac{D_{0+}^{\alpha-1} R(x, \lambda)(t)}{1+t^{\alpha}}\right| \\
& =\sup _{t \in[0,+\infty)}\left|\int_{0}^{t} \frac{1}{1+t^{\alpha}} h(s) d s\right| \\
& \leq m \sup _{t \in[0,+\infty)} \int_{0}^{t} \frac{1}{1+t^{\alpha}} d s \leq m,
\end{aligned}
$$


and

$$
\left\|D_{0+}^{\alpha} R(x, \lambda)(t)\right\|_{\infty}=\sup _{t \in[0,+\infty)}\left|D_{0+}^{\alpha} R(x, \lambda)(t)\right|=\sup _{t \in[0,+\infty)}|h(t)| \leq m .
$$

That is, $R(x, \lambda)(\bar{\Omega})$ is uniformly bounded in $X$. Next, we show that $R(x, \lambda)(\bar{\Omega})$ is equicontinuous on any compact interval of $[0,+\infty)$. In fact, for any $K>0, t_{1}, t_{2} \in[0, K], x \in \bar{\Omega}$, $\lambda \in[0,1]$, we have

$$
\begin{aligned}
& \left|\frac{R(x, \lambda)\left(t_{1}\right)}{1+t_{1}^{\alpha}}-\frac{R(x, \lambda)\left(t_{2}\right)}{1+t_{2}^{\alpha}}\right| \\
& \quad=\frac{1}{\Gamma(\alpha)}\left[\left|\int_{0}^{t_{1}} \frac{\left(t_{1}-s\right)^{\alpha-1}}{1+t_{1}^{\alpha}} h(s) d s-\int_{0}^{t_{2}} \frac{\left(t_{2}-s\right)^{\alpha-1}}{1+t_{2}^{\alpha}} h(s) d s\right|\right] \\
& \quad \leq \frac{1}{\Gamma(\alpha)}\left[\left|\int_{0}^{t_{1}}\left(\frac{\left(t_{1}-s\right)^{\alpha-1}}{1+t_{1}^{\alpha}}-\frac{\left(t_{2}-s\right)^{\alpha-1}}{1+t_{2}^{\alpha}}\right) h(s) d s\right|+\left|\int_{t_{1}}^{t_{2}} \frac{\left(t_{2}-s\right)^{\alpha-1}}{1+t_{2}^{\alpha}} h(s) d s\right|\right] \\
& \quad \leq \frac{m}{\Gamma(\alpha)}\left[\int_{0}^{t_{1}}\left|\frac{\left(t_{1}-s\right)^{\alpha-1}}{1+t_{1}^{\alpha}}-\frac{\left(t_{2}-s\right)^{\alpha-1}}{1+t_{2}^{\alpha}}\right| d s+\int_{t_{1}}^{t_{2}} \frac{\left(t_{2}-s\right)^{\alpha-1}}{1+t_{2}^{\alpha}} d s\right] \\
& \quad \leq \frac{m}{\Gamma(\alpha)}\left[\int_{0}^{t_{1}}\left|\frac{\left(t_{1}-s\right)^{\alpha-1}}{1+t_{1}^{\alpha}}-\frac{\left(t_{2}-s\right)^{\alpha-1}}{1+t_{2}^{\alpha}}\right| d s+\frac{1}{\alpha} \frac{\left(t_{2}-t_{1}\right)^{\alpha}}{1+t_{2}^{\alpha}}\right] \rightarrow 0 \quad \text { as } t_{1} \rightarrow t_{2} .
\end{aligned}
$$

So, $\left\{\frac{R(x, \lambda)(t)}{1+t^{\alpha}}, x \in \bar{\Omega}\right\}$ is equicontinuous on $[0, K]$. Similarly, we obtain that $\left\{\frac{D_{0+1}^{\alpha-1} R(x, \lambda)(t)}{1+t^{\alpha}}, x \in \bar{\Omega}\right\}$ is equicontinuous on $[0, K]$. In addition, since

$$
\begin{aligned}
\left|D_{0+}^{\alpha} R(x, \lambda)\left(t_{1}\right)-D_{0+}^{\alpha} R(x, \lambda)\left(t_{2}\right)\right| & =\left|h\left(t_{1}\right)-h\left(t_{2}\right)\right| \\
& =\left|\phi_{q}\left(l\left(t_{1}, x, \lambda\right)\right)-\phi_{q}\left(l\left(t_{2}, x, \lambda\right)\right)\right|
\end{aligned}
$$

and

$$
\begin{aligned}
|l(t, x, \lambda)| & =\left|\int_{t}^{+\infty}(Q-I) N_{\lambda} x(s) d s+\phi_{p}\left(D_{0+}^{\alpha} x(+\infty)\right)\right| \\
& \leq \tilde{r}+\phi_{p}(r) \quad(t \in[0, K], x \in \bar{\Omega}),
\end{aligned}
$$

we have

$$
\left|l\left(t_{1}, x, \lambda\right)-l\left(t_{2}, x, \lambda\right)\right|=\left|\int_{t_{1}}^{t_{2}}(Q-I) N_{\lambda} x(s) d s\right| \leq \int_{t_{1}}^{t_{2}}\left(g_{r}(s)+\frac{\left\|g_{r}\right\|_{L^{1}}}{\sum_{i=1}^{n} \alpha_{i} e^{-\xi_{i}}} e^{-s}\right) d s .
$$

By the absolute continuity of the integral, $\{l(t, x, \lambda), x \in \bar{\Omega}\}$ is equicontinuous on $[0, K]$, which combined with the uniform continuity of $\phi_{q}(x)$ on $\left[-\tilde{r}-\phi_{p}(r), \tilde{r}+\phi_{p}(r)\right]$, gives that $\left\{D_{0+}^{\alpha} R(x, \lambda)(t), x \in \bar{\Omega}\right\}$ is equicontinuous on $[0, K]$.

Step 2 . We establish the fact that $R(x, \lambda)(\bar{\Omega})$ is equiconvergent at infinity. In fact, for any $x \in \bar{\Omega}$, by (3.4) we have

$$
\lim _{s \rightarrow+\infty} \int_{s}^{+\infty}(Q-I) N_{\lambda} x(\tau) d \tau=0
$$


Since $\phi_{q}(x)$ is uniformly continuous on $\left[-\tilde{r}-\phi_{p}(r), \tilde{r}+\phi_{p}(r)\right]$, for any $\varepsilon>0$, there exists a constant $L_{1}>0$ such that, for $s \geq L_{1}$, we have

$$
\begin{aligned}
|h(s)| & =\left|\phi_{q}\left[\int_{s}^{+\infty}(Q-I) N_{\lambda} x(\tau) d \tau+\phi_{p}\left(D_{0+}^{\alpha} x(+\infty)\right)\right]-\phi_{q}\left[\phi_{p}\left(D_{0+}^{\alpha} x(+\infty)\right)\right]\right| \\
& <\varepsilon, \quad \forall x \in \bar{\Omega} .
\end{aligned}
$$

Therefore, $|h(s)|<\varepsilon$ for $s \geq L_{1}$ and $|h(s)| \leq m$ for $s<L_{1}$. On the other hand, since $\lim _{t \rightarrow+\infty} \frac{t^{\alpha-1}}{1+t^{\alpha}}=0$ and $\lim _{t \rightarrow+\infty} \frac{1}{1+t^{\alpha}}=0$ for the above $\varepsilon>0$, there exists a constant $L>L_{1}>0$ such that, for any $t_{1}, t_{2} \geq L$ and $0 \leq s \leq L_{1}$, we have

$$
\left|\frac{\left(t_{1}-s\right)^{\alpha-1}}{1+t_{1}^{\alpha}}-\frac{\left(t_{2}-s\right)^{\alpha-1}}{1+t_{2}^{\alpha}}\right| \leq \frac{t_{1}^{\alpha-1}}{1+t_{1}^{\alpha}}+\frac{t_{2}^{\alpha-1}}{1+t_{2}^{\alpha}}<\varepsilon
$$

and

$$
\left|\frac{1}{1+t_{1}^{\alpha}}-\frac{1}{1+t_{2}^{\alpha}}\right| \leq \frac{1}{1+t_{1}^{\alpha}}+\frac{1}{1+t_{2}^{\alpha}}<\varepsilon
$$

Then, for $t_{1}, t_{2} \geq L$, from the above we obtain

$$
\begin{aligned}
& \left|\frac{R(x, \lambda)\left(t_{1}\right)}{1+t_{1}^{\alpha}}-\frac{R(x, \lambda)\left(t_{2}\right)}{1+t_{2}^{\alpha}}\right| \\
& =\frac{1}{\Gamma(\alpha)}\left|\int_{0}^{t_{1}} \frac{\left(t_{1}-s\right)^{\alpha-1}}{1+t_{1}^{\alpha}} h(s) d s-\int_{0}^{t_{2}} \frac{\left(t_{2}-s\right)^{\alpha-1}}{1+t_{2}^{\alpha}} h(s) d s\right| \\
& \leq \frac{1}{\Gamma(\alpha)} \int_{0}^{L_{1}}\left|\frac{\left(t_{1}-s\right)^{\alpha-1}}{1+t_{1}^{\alpha}}-\frac{\left(t_{2}-s\right)^{\alpha-1}}{1+t_{2}^{\alpha}}\right| h(s)\left|d s+\frac{1}{\Gamma(\alpha)} \int_{L_{1}}^{t_{1}} \frac{\left(t_{1}-s\right)^{\alpha-1}}{1+t_{1}^{\alpha}}\right| h(s) \mid d s \\
& \quad+\frac{1}{\Gamma(\alpha)} \int_{L_{1}}^{t_{2}} \frac{\left(t_{2}-s\right)^{\alpha-1}}{1+t_{2}^{\alpha}}|h(s)| d s \\
& \leq \frac{m}{\Gamma(\alpha)} L_{1} \varepsilon+\frac{2 m}{\Gamma(\alpha+1)} \varepsilon .
\end{aligned}
$$

Similarly, we get

$$
\left|\frac{D_{0+}^{\alpha-1} R(x, \lambda)\left(t_{1}\right)}{1+t_{1}^{\alpha}}-\frac{D_{0+}^{\alpha-1} R(x, \lambda)\left(t_{2}\right)}{1+t_{2}^{\alpha}}\right| \leq m L_{1} \varepsilon+2 \varepsilon
$$

and, in addition,

$$
\begin{aligned}
\left|D_{0+}^{\alpha} R(x, \lambda)\left(t_{1}\right)-D_{0+}^{\alpha} R(x, \lambda)\left(t_{2}\right)\right| & =\left|h\left(t_{1}\right)-h\left(t_{2}\right)\right| \\
& \leq\left|h\left(t_{1}\right)\right|+\left|h\left(t_{2}\right)\right| \\
& <2 \varepsilon .
\end{aligned}
$$

So, $R(x, \lambda)(\bar{\Omega})$ is equiconvergent at infinity. By Lemma 3.2, $R: \bar{\Omega} \times[0,1] \rightarrow X_{2}$ is completely continuous. 
Finally, we prove that the $\left(a_{3}\right)$ and $\left(a_{4}\right)$ in Definition 2.4 hold. Let $x \in \sum_{\lambda}=\{x \in \bar{\Omega} \mid M x=$ $\left.N_{\lambda} x\right\}$. Then $\left(\phi_{p}\left(D_{0+}^{\alpha} x(t)\right)\right)^{\prime}=N_{\lambda} x(t) \in \operatorname{Im} M=\operatorname{Ker} Q$ and

$$
\begin{aligned}
R(x, \lambda)(t)= & I_{0+}^{\alpha} h(t) \\
= & \frac{1}{\Gamma(\alpha)} \int_{0}^{t}(t-s)^{\alpha-1}\left[\phi_{q}\left(\int_{s}^{+\infty}(Q-I) N_{\lambda} x(\tau) d \tau+\phi_{p}\left(D_{0+}^{\alpha} x(+\infty)\right)\right)\right. \\
& \left.-D_{0+}^{\alpha} x(+\infty)\right] d s \\
= & \frac{1}{\Gamma(\alpha)} \int_{0}^{t}(t-s)^{\alpha-1} \phi_{q}\left(\int_{s}^{+\infty}-N_{\lambda} x(\tau) d \tau+\phi_{p}\left(D_{0+}^{\alpha} x(+\infty)\right)\right) d s-(P x)(t) \\
= & \frac{1}{\Gamma(\alpha)} \int_{0}^{t}(t-s)^{\alpha-1} \phi_{q}\left(\int_{s}^{+\infty}-\left(\phi_{p}\left(D_{0+}^{\alpha} x(\tau)\right)\right)^{\prime} d \tau+\phi_{p}\left(D_{0+}^{\alpha} x(+\infty)\right)\right) d s \\
& -(P x)(t) \\
= & \frac{1}{\Gamma(\alpha)} \int_{0}^{t}(t-s)^{\alpha-1} D_{0+}^{\alpha} x(s) d s-(P x)(t),
\end{aligned}
$$

which, combined with boundary conditions, yields that

$$
R(x, \lambda)(t)=x(t)-(P x)(t)=[(I-P) x](t)
$$

It is clear that $R(x, 0)(t)$ is a zero operator, and for any $x \in \bar{\Omega}$, we have

$$
\begin{aligned}
M & {[P x+R(x, \lambda)](t) } \\
& =M\left[\frac{1}{\Gamma(\alpha)} \int_{0}^{t}(t-s)^{\alpha-1} \phi_{q}\left(\int_{s}^{+\infty}(Q-I) N_{\lambda} x(\tau) d \tau+\phi_{p}\left(D_{0+}^{\alpha} x(+\infty)\right)\right) d s\right] \\
& =\left[\int_{t}^{+\infty}(Q-I) N_{\lambda} x(s) d s+\phi_{p}\left(D_{0+}^{\alpha} x(+\infty)\right)\right]^{\prime} \\
& =(I-Q) N_{\lambda} x(t) .
\end{aligned}
$$

By the above, $N_{\lambda}$ is $M$-compact on $\bar{\Omega}$.

Theorem 3.1 Suppose that $\left(A_{1}\right)$ and the following conditions hold:

$\left(A_{2}\right)$ there exist nonnegative functions $a(t), b(t), c(t), d(t) \in Y$ such that

$$
\begin{aligned}
|f(t, u, v, w)| \leq & a(t)+b(t) \frac{|u|^{p-1}}{\left(1+t^{\alpha}\right)^{p-1}}+c(t) \frac{|v|^{p-1}}{\left(1+t^{\alpha}\right)^{p-1}} \\
& +d(t)|w|^{p-1}, \quad \forall t \in[0,+\infty),(u, v, w) \in \mathbb{R}^{3}
\end{aligned}
$$

$\left(A_{3}\right)$ there exists a positive constant B such that one of the following inequalities hold:

$$
\begin{aligned}
& w f(t, u, v, w)>0, \quad \forall t \in[0,+\infty), u, v \in \mathbb{R},|w|>B, \\
& w f(t, u, v, w)<0, \quad \forall t \in[0,+\infty), u, v \in \mathbb{R},|w|>B .
\end{aligned}
$$

Then BVP (1.1) has at least one solution in $X$, provided that $\|b\|_{1}+\|c\|_{1}+\|d\|_{1}<1$. 
Before we prove Theorem 3.1, we show two lemmas.

Lemma 3.4 Let $\Omega_{1}=\left\{x \in \operatorname{dom} M \backslash \operatorname{Ker} M \mid M x=N_{\lambda} x, \lambda \in(0,1)\right\}$. Suppose that $\left(A_{2}\right)$ and $\left(A_{3}\right)$ hold. Then $\Omega_{1}$ is bounded in $X$.

Proof Let $x \in \Omega_{1}$. Then $M x=N_{\lambda} x$ and thus $Q N_{\lambda} x=0$, that is,

$$
\sum_{i=1}^{n} \alpha_{i} \int_{\xi_{i}}^{+\infty} f\left(s, x(s), D_{0+}^{\alpha-1} x(s), D_{0+}^{\alpha} x(s)\right) d s=0
$$

By Lemma 2.1 and the boundary conditions we have

$$
x(t)=c_{1} t^{\alpha-1}+c_{2} t^{\alpha-2}+I_{0+}^{\alpha} D_{0+}^{\alpha} x(t)=I_{0+}^{\alpha} D_{0+}^{\alpha} x(t)=\frac{1}{\Gamma(\alpha)} \int_{0}^{t}(t-s)^{\alpha-1} D_{0+}^{\alpha} x(s) d s .
$$

Thus,

$$
\|x\|_{0} \leq\left\|D_{0_{+}}^{\alpha} x\right\|_{\infty}, \quad\left\|D_{0+}^{\alpha-1} x\right\|_{0} \leq\left\|D_{0_{+}}^{\alpha} x\right\|_{\infty}
$$

By $\left(A_{3}\right)$ there exists a constant $s_{0} \in[0,+\infty)$ such that $\left|D_{0+}^{\alpha} x\left(s_{0}\right)\right| \leq B$, which, combined with $M x=N_{\lambda} x$ and $\left(A_{2}\right)$, gives

$$
\begin{aligned}
& \left|\phi_{p}\left(D_{0+}^{\alpha} x(t)\right)\right| \\
& \quad=\left|\phi_{p}\left(D_{0+}^{\alpha} x\left(s_{0}\right)\right)-\int_{s_{0}}^{t} \lambda f\left(s, x(s), D_{0+}^{\alpha-1} x(s), D_{0+}^{\alpha} x(s)\right) d s\right| \\
& \quad \leq\left|\phi_{p}\left(D_{0+}^{\alpha} x\left(s_{0}\right)\right)\right|+\left|\int_{s_{0}}^{t} \lambda f\left(s, x(s), D_{0+}^{\alpha-1} x(s), D_{0+}^{\alpha} x(s)\right) d s\right| \\
& \quad \leq \phi_{p}(B)+\int_{0}^{+\infty}\left|f\left(s, x(s), D_{0+}^{\alpha-1} x(s), D_{0+}^{\alpha} x(s)\right)\right| d s \\
& \quad \leq \phi_{p}(B)+\int_{0}^{+\infty}\left[a(s)+b(s) \frac{|x|^{p-1}}{\left(1+s^{\alpha}\right)^{p-1}}+c(s) \frac{\left|D_{0+}^{\alpha-1} x\right|^{p-1}}{\left(1+s^{\alpha}\right)^{p-1}}+d(s)\left|D_{0+}^{\alpha} x\right|^{p-1}\right] d s \\
& \quad \leq \phi_{p}(B)+\|a\|_{1}+\|b\|_{1} \phi_{p}\left(\|x\|_{0}\right)+\|c\|_{1} \phi_{p}\left(\left\|D_{0+}^{\alpha-1} x\right\|_{0}\right)+\|d\|_{1} \phi_{p}\left(\left\|D_{0+}^{\alpha} x\right\|_{\infty}\right) \\
& \quad \leq \phi_{p}(B)+\|a\|_{1}+\left[\|b\|_{1}+\|c\|_{1}+\|d\|_{1}\right] \phi_{p}\left(\left\|D_{0+}^{\alpha} x\right\|_{\infty}\right) .
\end{aligned}
$$

Then

$$
\begin{aligned}
\left\|\phi_{p}\left(D_{0+}^{\alpha} x\right)\right\|_{\infty} & =\phi_{p}\left(\left\|D_{0+}^{\alpha} x\right\|_{\infty}\right) \\
& \leq \phi_{p}(B)+\|a\|_{1}+\left[\|b\|_{1}+\|c\|_{1}+\|d\|_{1}\right] \phi_{p}\left(\left\|D_{0+}^{\alpha} x\right\|_{\infty}\right) .
\end{aligned}
$$

Thus,

$$
\phi_{p}\left(\left\|D_{0+}^{\alpha} x\right\|_{\infty}\right) \leq \frac{\phi_{p}(B)+\|a\|_{1}}{1-\left[\|b\|_{1}+\|c\|_{1}+\|d\|_{1}\right]}:=A .
$$

That is,

$$
\left\|D_{0+}^{\alpha} x\right\|_{\infty} \leq \phi_{q}(A) .
$$


Therefore,

$$
\|x\|_{X}=\max \left\{\|x\|_{0},\left\|D_{0+}^{\alpha-1} x\right\|_{0},\left\|D_{0+}^{\alpha} x\right\|_{\infty}\right\} \leq \phi_{q}(A)
$$

So, $\Omega_{1}$ is bounded in $X$.

Lemma 3.5 Let $\Omega_{2}=\{x \in \operatorname{Ker} M \mid N x \in \operatorname{Im} M\}$. Suppose that $\left(A_{3}\right)$ holds. Then $\Omega_{2}$ is bounded in $X$.

Proof Let $x \in \Omega_{2}$, that is, $x=c t^{\alpha}, c \in \mathbb{R}, Q N x=0$, so that

$$
\sum_{i=1}^{n} \alpha_{i} \int_{\xi_{i}}^{+\infty} f\left(t, c t^{\alpha}, c \Gamma(\alpha+1) t, c \Gamma(\alpha)\right) d t=0
$$

By $\left(A_{3}\right)$ we have $|c \Gamma(\alpha)| \leq B$, that is, $|c| \leq \frac{B}{\Gamma(\alpha)}$. Therefore,

$$
\begin{aligned}
\|x\|_{X} & =\max \left\{\|x\|_{0},\left\|D_{0+}^{\alpha-1} x\right\|_{0},\left\|D_{0+}^{\alpha} x\right\|_{\infty}\right\} \\
& =\max \left\{\left\|\frac{c t^{\alpha}}{1+t^{\alpha}}\right\|_{\infty},\left\|\frac{c \Gamma(\alpha+1) t}{1+t^{\alpha}}\right\|_{\infty},|c \Gamma(\alpha)|\right\} \\
& \leq\left\|\frac{c t^{\alpha}}{1+t^{\alpha}}\right\|_{\infty}+\left\|\frac{c \Gamma(\alpha+1) t}{1+t^{\alpha}}\right\|_{\infty}+|c \Gamma(\alpha)| \leq(1+\Gamma(\alpha)+\Gamma(\alpha+1))|c|:=C .
\end{aligned}
$$

So, $\Omega_{2}$ is bounded in $X$.

Proof of Theorem 3.1 Set $\Omega=\left\{x \in X \mid\|x\|_{X}<\max \left\{B, \phi_{q}(A), C\right\}+1\right\}$. By Lemma 3.1 and Lemma 3.3 we know that $M$ is quasi-linear and $N_{\lambda}$ is $M$-compact on $\bar{\Omega}$. From Lemma 3.4 and Lemma 3.5 we obtain:

$\left(B_{1}\right) \quad M x \neq N_{\lambda} x, \forall(x, \lambda) \in \partial \Omega \times(0,1)$,

$\left(B_{2}\right) Q N x \neq 0, \forall x \in \operatorname{Ker} M \cap \partial \Omega$.

Now we show $\left(B_{3}\right)$ holds. Let $J: \operatorname{Im} Q \rightarrow \operatorname{Ker} M$ be the homeomorphism defined by

$$
J\left(c e^{-t}\right)=c t^{\alpha}, \quad c \in \mathbb{R}, t \in[0,+\infty) .
$$

Without loss of generality, we suppose that (3.6) holds. Define the homotopic mapping

$$
H(x, \lambda)=\lambda x-(1-\lambda) J Q N x, \quad \forall x \in \bar{\Omega} \cap \operatorname{Ker} M, \lambda \in[0,1] .
$$

Then $H(x, \lambda) \neq 0, x \in \partial \Omega \cap \operatorname{Ker} M, \lambda \in[0,1]$. Indeed, for $x \in \partial \Omega \cap \operatorname{Ker} M$, we have $x=c t^{\alpha}$ and thus

$$
H(x, \lambda)=\lambda c t^{\alpha}-(1-\lambda) \frac{\sum_{i=1}^{n} \alpha_{i} \int_{\xi_{i}}^{+\infty} f\left(s, c s^{\alpha}, c \Gamma(\alpha+1) s, c \Gamma(\alpha)\right) d s}{\sum_{i=1}^{n} \alpha_{i} e^{-\xi_{i}}} t^{\alpha} .
$$

Clearly, $H(x, 1) \neq 0, x \in \partial \Omega \cap \operatorname{Ker} M$. For $\lambda \in[0,1)$ and $x=c t^{\alpha} \in \partial \Omega \cap \operatorname{Ker} M$, if $H(x, \lambda)=0$, then

$$
\frac{\sum_{i=1}^{n} \alpha_{i} \int_{\xi_{i}}^{+\infty} c \Gamma(\alpha) f\left(s, c s^{\alpha}, c \Gamma(\alpha+1) s, c \Gamma(\alpha)\right) d s}{\sum_{i=1}^{n} \alpha_{i} e^{-\xi_{i}}}=\frac{\lambda}{1-\lambda} c^{2} \Gamma(\alpha) \geq 0,
$$


which contradicts (3.6). If (3.5) holds, then defining the homotopic mapping

$$
H(x, \lambda)=\lambda x+(1-\lambda) J Q N x, \quad x \in \bar{\Omega} \cap \operatorname{Ker} M, \lambda \in[0,1],
$$

we also get contradiction in a similar way. Therefore, via the homotopy property of degree, we obtain

$$
\begin{aligned}
\operatorname{deg}(U Q N, \Omega \cap \operatorname{Ker} M, 0) & =\operatorname{deg}(H(\cdot, 0), \Omega \cap \operatorname{Ker} M, 0) \\
& =\operatorname{deg}(H(\cdot, 1), \Omega \cap \operatorname{Ker} M, 0) \\
& =\operatorname{deg}(I, \Omega \cap \operatorname{Ker} M, 0) \neq 0 .
\end{aligned}
$$

Applying Lemma 2.2, we conclude that (1.1) has at least one solution in $\bar{\Omega}$.

\section{Example}

Example 4.1 Consider the BVP

$$
\left\{\begin{array}{l}
\left(\phi_{3 / 2}\left(D_{0+}^{3 / 2} x(t)\right)\right)^{\prime}+\frac{e^{-3 t}}{4 \sqrt{1+t^{3 / 2}}} \sin \sqrt{|x(t)|}+\frac{1}{2} e^{-t} \phi_{3 / 2}\left(D_{0+}^{3 / 2} x(t)\right)+\frac{1}{4} e^{-3 t}=0, \\
0<t<+\infty, \\
x(0)=x^{\prime}(0)=0, \quad \phi_{3 / 2}\left(D_{0+}^{3 / 2} x(+\infty)\right)=\sum_{i=1}^{n} \alpha_{i} \phi_{3 / 2}\left(D_{0+}^{3 / 2} x\left(\xi_{i}\right)\right),
\end{array}\right.
$$

where $0<\xi_{1}<\xi_{2}<\cdots<\xi_{n}<+\infty, \alpha_{i}>0, \sum_{i=1}^{n} \alpha_{i}=1$. Let

$$
\begin{aligned}
& a(t)=b(t)=\frac{1}{4} e^{-3 t}, \quad c(t)=0, \quad d(t)=\frac{1}{2} e^{-t}, \quad B=4, \\
& g_{r}(t)=\left(\frac{1}{4} e^{-3 t}+\frac{1}{2} e^{-t}\right) r+\frac{1}{4} e^{-3 t} .
\end{aligned}
$$

We easily check $\left(A_{1}\right)-\left(A_{3}\right)$. By Theorem 3.1, problem (4.1) has at least one solution.

Competing interests

The authors declare that they have no competing interests.

\section{Authors' contributions}

The authors have made equal contributions to each part of this paper. All the authors read and approved the final manuscript.

\section{Acknowledgements}

This research is supported by the National Natural Science Foundation of China (No. 11271364).

Received: 2 March 2016 Accepted: 26 May 2016 Published online: 07 July 2016

\section{References}

1. Agarwal, RP, O'Regan, D: Infinite Interval Problems for Differential, Difference and Integral Equations. Kluwer Academic, Dordrecht (2001)

2. Gomes, JM, Sanchez, L: A variational approach to some boundary value problems in the half-line. Z. Angew. Math. Phys. 56(2), 192-209 (2005)

3. Arara, A, Benchohra, M, Hamidi, N, et al.: Fractional order differential equations on an unbounded domain. Nonlinear Anal., Theory Methods Appl. 72(2), 580-586 (2010)

4. Lian, H, Pang, H, Ge, W: Triple positive solutions for boundary value problems on infinite intervals. Nonlinear Anal., Theory Methods Appl. 67(7), 2199-2207 (2007)

5. Liu, Y: Existence and unboundedness of positive solutions for singular boundary value problems on half-line. Appl. Math. Comput. 144(2), 543-556 (2003)

6. Su, X, Zhang, S: Unbounded solutions to a boundary value problem of fractional order on the half-line. Comput. Math. Appl. 61(4), 1079-1087 (2011) 
7. Tian, Y, Ge, W, Shan, W: Positive solutions for three-point boundary value problem on the half-line. Comput. Math. Appl. 53(7), 1029-1039 (2007)

8. Zhao, $\mathrm{X}, \mathrm{Ge}, \mathrm{W}$ : Unbounded solutions for a fractional boundary value problems on the infinite interval. Acta Appl. Math. 109(2), 495-505 (2010)

9. Chen, SZ, Zhang, Y: Singular boundary value problems on a half-line. J. Math. Anal. Appl. 195(2), 449-468 (1995)

10. Liu, X, Jia, M: Multiple solutions of nonlocal boundary value problems for fractional differential equations on the half-line. Electron. J. Qual. Theory Differ. Equ. 2011, 56 (2011)

11. Yan, B: Multiple unbounded solutions of boundary value problems for second-order differential equations on the half-line. Nonlinear Anal., Theory Methods Appl. 51(6), 1031-1044 (2002)

12. Yan, B, O'Regan, D, Agarwal, RP: Unbounded solutions for singular boundary value problems on the semi-infinite interval: upper and lower solutions and multiplicity. J. Comput. Appl. Math. 197(2), 365-386 (2006)

13. Jiang, W: Solvability for $p$-Laplacian boundary value problem at resonance on the half-line. Bound. Value Probl. 2013(1), $207(2013)$

14. Lian, H, Pang, H, Ge, W: Solvability for second-order three-point boundary value problems at resonance on a half-line J. Math. Anal. Appl. 337(2), 1171-1181 (2008)

15. Zhou, H, Kou, C, Xie, F: Existence of solutions for fractional differential equations with multi-point boundary conditions at resonance on a half-line. Electron. J. Qual. Theory Differ. Equ. 2011, 27 (2011)

16. Ge, W, Ren, J: An extension of Mawhin's continuation theorem and its application to boundary value problems with a p-Laplacian. Nonlinear Anal., Theory Methods Appl. 58(3), 477-488 (2004)

17. Wang, J, Ibrahim, AG, Feckan, M: Nonlocal impulsive fractional differential inclusions with fractional sectorial operators on Banach spaces. Appl. Math. Comput. 257(2015), 103-118 (2015)

18. Wang, J, Zhou, Y, Feckan, M: Nonlinear impulsive problems for fractional differential equations and Ulam stability. Comput. Math. Appl. 64(10), 3389-3405 (2012)

19. Kilbas, AA, Srivastava, HM, Trujillo, JJ: Theory and Applications of Fractional Differential Equations. Elsevier, Amsterdam (2006)

20. Miller, KS, Ross, B: An Introduction to the Fractional Calculus and Fractional Differential Equations. Wiley, New York (1993)

\section{Submit your manuscript to a SpringerOpen ${ }^{\circ}$ journal and benefit from:}

- Convenient online submission

Rigorous peer review

- Immediate publication on acceptance

Open access: articles freely available online

- High visibility within the field

- Retaining the copyright to your article 